\title{
Green Synthesis of Silver Nanoparticles Using Solar Radiation and Loeselia Mexicana Leaf Extract
}

\author{
R. Herrero-Calvillo ${ }^{1}$, S. E. Borjas-García ${ }^{2}$, G. Rosas $^{1 *}$ \\ 1. Instituto de Investigación en Metalurgia y Materiales, UMSNH, Morelia Michoacán, Mexico. \\ 2. Instituto de Física y Matemáticas, UMSNH, Morelia Michoacán, Mexico. \\ * Corresponding author: grtrejo@umich.mx
}

Currently, nanomaterials have received significant attention from researchers of several disciplines due to the wide variety of technological applications. The noble metal nanoparticles have a wide variety of applications in the area of medicine, electronic devices, catalysis, antibacterial materials, among others [1]. Within the wide variety of nanoparticles, silver ones stand out due to their high catalytic and electronic properties, in addition to being difficult to oxidize, which makes them easier to obtain than other nanoparticles of different nature [2]. One of the synthesis methods that has obtained much relevance is the green synthesis, in which the production of metallic nanoparticles is achieved by taking advantage of the reducing and capping properties of the extracted compounds from biological systems, such as plants, fungi and other natural compounds $[3,4]$.

In this work, Loeselia Mexicana leaf extract was used. Fresh Loeselia Mexicana leaves were disjointed from the stems to be dried under room temperature, milled and washed with distilled water using magnetic stirring. Biomolecules extraction was carried out by mixing the leaf powder with $100 \mathrm{ml}$ of distilled water at $70^{\circ} \mathrm{C}$ for 10 minutes using magnetic stirring. The leaf extract was filtered and cooled at room temperature. Three Ag nanoparticles samples were obtained by mixing the leaf extract with $2 \mathrm{mM}$ $\mathrm{AgNO}_{3}$ in a 1:1 volume ratio. The three samples were exposed under solar radiation for $0.75,30$ and 60 min. Synthesized samples showed a color change from pale yellow to reddish brown, indicating that silver nanoparticles were obtained. Nanoparticles growth and stabilization were monitored by UV-Vis spectroscopy (OceanOptics USB 4000). The XRD pattern was obtained in a Bruker D8 Advance, DAVINCI Lynx eye diffractometer. Furthermore, SEM images were obtained by backscattered electron technique (JEOL JSM-7600F).

Figure 1 shows the silver nanoparticles UV-Vis spectra, the peaks are located between 400-500 $\mathrm{nm}$ in all the samples, and this range is characteristic of the silver nanoparticles. The sample obtained in $0.75 \mathrm{~min}$ showed a very faint color change and a low absorbance SPR peak. After 30 min, a defined SPR peak was obtained with greater absorbance value. Besides, the $60 \mathrm{~min}$ sample showed a higher absorbance SPR peak; however, the peak widened considerably concerning the previous sample, indicating an increase in the nanoparticles size distribution. Figure 2 shows the XRD diffractogram, in which the formation of $\mathrm{Ag}$ nanoparticles, together with $\mathrm{AgCl}$ as crystals byproducts of the reaction, is observed. Figure 3 corresponds to the SEM images of the Ag nanoparticles. It can be seen that the $0.75 \mathrm{~min}$ sample (Figure $3 a$ ) contains a tiny amount of nanometric particles. Figure $3 b$, corresponding to the 30 min sample, shows that it contains a much larger amount of nanoparticles with a homogeneous size and shape. On the other hand, the 60 min sample (figure 3c), contains a greater quantity of nanoparticles; however, it can be seen that there is a high size distribution. Solar radiation directly affects the Ag nanoparticles formation; nevertheless, an excess of exposure time generates an uncontrolled nanoparticles formation of different size ranges. The exposure time should be around $30 \mathrm{~min}$, in order to obtain homogeneous morphology and size nanoparticles. 


\section{References:}

[1] RD Rivera-Rangel et al., Colloids and Surfaces A: Physicochemical and Engineering Aspects 536 (2018), p. 60.

[2] V Soshnikova et al., Artificial cells, nanomedicine, and biotechnology 46 (2018), p. 108.

[3] D-Y Kim et al., Journal of Cleaner Production 172 (2018), p. 2910.

[4] Y Huo et al., Artificial cells, nanomedicine, and biotechnology 46 (2018), p. 303.

[5] The authors acknowledge funding from H. Consejo Nacional de Ciencia y Tecnología (CONACyT), México.

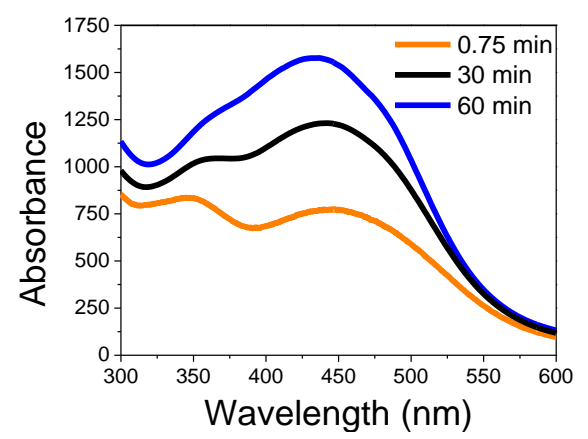

Figure 1. UV-Vis spectra of Ag nanoparticles synthesized with Loeselia Mexicana extract under sunlight.

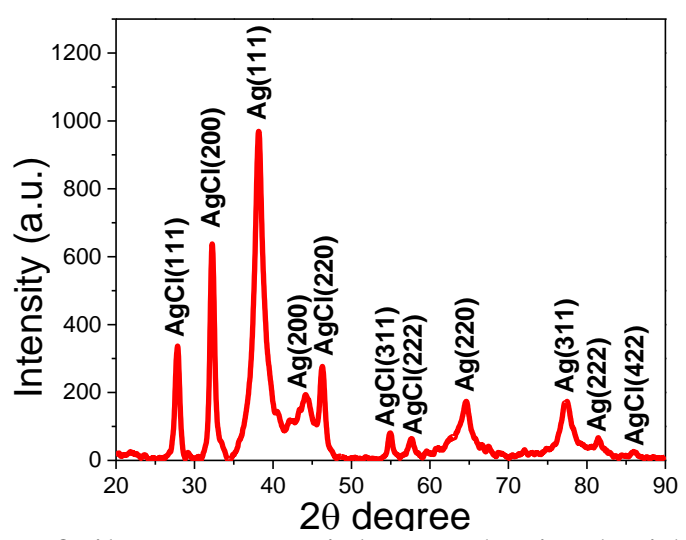

Figure 2. X-Ray diffractogram of silver nanoparticles synthesized with Loeselia Mexicana extract.
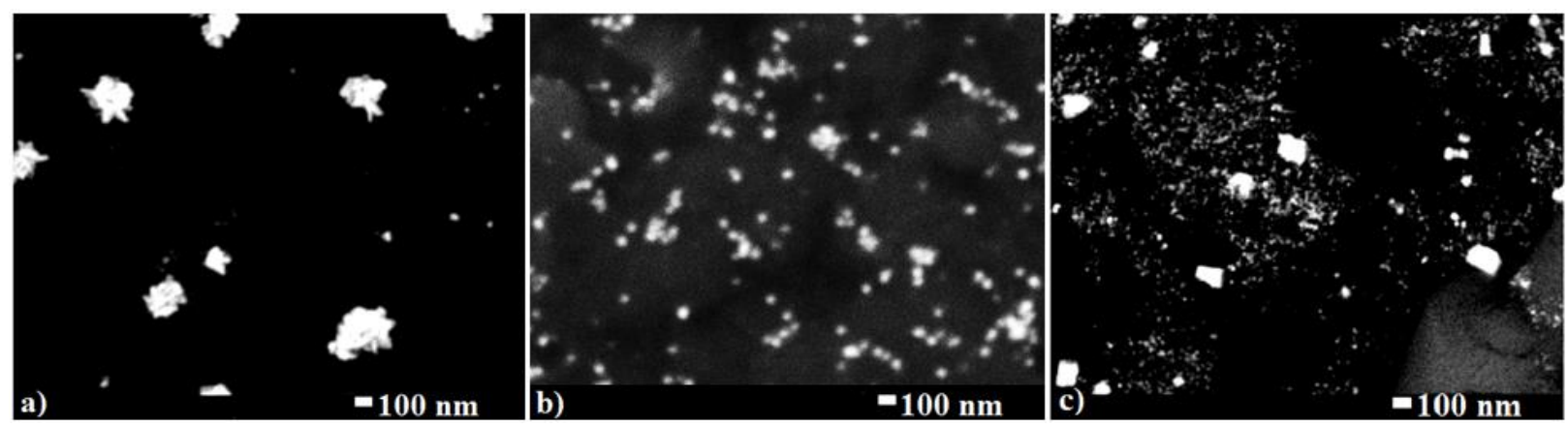

Figure 3. SEM images of silver nanoparticles synthesized with Loeselia Mexicana extract under sunlight: (a) 0.75, (b) 30, and (c) $60 \mathrm{~min}$. 